\title{
Alteration of Ferritin Levels and Lymphocytes Counts in Saudi Patients with COVID-19 Infection in Al Madinah Al Munawarah
}

\author{
Walaa Mohammedsaeed ${ }^{1, *}$, Amal M. Surrati ${ }^{2}$, Hadiah Q. Alnakhli ${ }^{3}$, Malak Alharbi ${ }^{4}$ Nidda Syeed $^{1}$ \\ ${ }^{1}$ Medical Laboratory Science, College of Applied Medical Sciences, Taibah University, Madinah, Saudi Arabia \\ ${ }^{2}$ Family and Community Medicine, College of Medicine, Taibah University, Madinah, Saudi Arabia \\ ${ }^{3}$ Department of Internal Medicine, Ohud Hospital, Madinah, Saudi Arabia \\ ${ }^{4}$ Department of Internal Medicine, Madinah General Hospital, Madinah, Saudi Arabia
}

Email address:

wmohammedsaeed@taibahu.edu.sa (W. Mohammedsaeed)

${ }^{*}$ Corresponding author

\section{To cite this article:}

Walaa Mohammedsaeed, Amal M. Surrati, Hadiah Q. Alnakhli, Malak Alharbi, Nidda Syeed. Alteration of Ferritin Levels and Lymphocytes Counts in Saudi Patients with COVID-19 Infection in Al Madinah Al Munawarah. International Journal of Diabetes and Endocrinology. Vol. 5, No. 4, 2020, pp. 61-66. doi: 10.11648/j.ijde.20200504.12

Received: November 12, 2020; Accepted: November 27, 2020; Published: December 4, 2020

\begin{abstract}
It is an essential to evaluate the potential biomarkers affecting the progression of disease in COVID-19 patients. Therefore, we investigated the relationship between Ferritin levels and hematological parameters and severity of COVID-19 to explore its role in predicting the severity of COVID-19. This retrospective study was conducted in different Hospitals at Madinah region, Saudi Arabia. In the present study, a total of 112 cases of COVID-19 patients. The patient records included the clinical and laboratory data that was used for analysis by using the GraphPad Prism Software. Data presented as mean (SD), all differences were statistically significant at the level of $\mathrm{P} \leq 0.05$ or $\leq 0.001$. There were significantly increased levels of ferritin, neutrophil and leukocyte counts in severe COVID-19 cases (1857 (50.9), 9.9 (2.8), 9.9 (2.8) respectively) compared with non-sever cases. Additionally, there were significantly reduction in lymphocytes counts in COVID-19 patients (1.3 (9.5), $\mathrm{P}=0.001)$. There were also strong correlations between elevation of ferritin levels or reduction of lymphocytes and the severity COVID-19 infection ( $\mathrm{r}=0.53$, OR: 5.88 and $\mathrm{r}=-0.55$, OR: 3.98 respectively). The results demonstrate that high values for neutrophil, leukocyte counts and Ferritin levels, and low values for lymphocyte counts may have diagnostic properties concerning COVID-19 in severe cases.
\end{abstract}

Keywords: Neutrophil, Leukocyte, Ferritin, Lymphocyte, COVID-19

\section{Introduction}

The coronavirus disease (COVID-19) is initiated by a large family of coronaviruses (CoV's) that are found varyingly in animal species. The COVID-19 has led to the outbreak of respiratory syndrome that noticed and reported in Wuhan, China in December 2019. Globally it has affected over 210 countries. The World Health Organization (WHO) declared it a Public Health Emergency of International Concern in January [1]. The infection spreads by human to human transmission. The symptoms include high fever, persistent dry cough, myalgia, pneumonia, headache, diarrhea. There has been prompt outpouring of research after the outbreak of COVID-19, on the epidemiology, clinical features, and diagnosis [1].

A study on early virus transmission has reported that the median age of patients is 59 years, between 15 to 89 years, with the majority (59\%) being male [2]. It was further reported that the people with weak immune system like older people and those with underlying medical condition like cardiovascular, diabetic, renal and hepatic dysfunction are at higher risk [3-5]. The association between clinical parameters and the severity of COVID-19 is crucial in understanding their role and in predicting the severity of disease. In the recent past, several clinical parameters have been studied that 
could help in distinguishing between mild and severe disease and possibly may be used as prognostic markers. One of the crucial laboratory investigations are the hematological parameters. A prior study indicated that white blood cell neutrophil count, lymphocyte count and albumin were risk factors for intensive care unit (ICU) care in patients with COVID-19 [6]. Similarly, hyperferritinemia or hyperferritinemia syndrome has been linked with increased illness and fatal outcomes. Hyperferritinemia, indicates the occurrence of viruses and bacteria in the body [7]. Ferritin is a main intracellular iron storage protein in all organisms. It combines free ions of the trace element, neutralizing its toxic properties and enhancing its solubility. In the soluble structure, the body can expend iron as required, in particular for regulation of cellular oxygen metabolism. Low ferritin levels result in decrease iron concentrations and iron deficiency anemia. Hyperferritinemia, is a disorder where high levels of intracellular iron storage protein, called ferritin are found, that lead to the activation of macrophages resulting in increased cytokine secretion [7, 8]. Cytokines are a type of signaling molecules that mediate and regulate immunity. At low concentrations, they are safe for the body and assist to defend it against viruses and bacteria [9]. At high levels, the so called "cytokine storm" develops, which can be deadly for half of the patients, particularly for the elderly $[8,9]$.

Therefore, it is an essential to assess the potential factors influencing the progression of disease in COVID-19 patients. We investigated the relationship between Ferritin levels and hematological indicators and severity of COVID-19 to explore its role in predicting the severity of COVID-19.

\section{Methods}

\subsection{Study Design and Data Collection}

This retrospective study was conducted in the Ohud Hospital and King Fahd Hospital of Madinah region, Saudi Arabia. In the current study, a total of 112 cases of COVID-19 patients. The patient files contained the clinical and laboratory data that was used for analysis. All cases were evaluated based on the inclusion and exclusion criteria; the inclusion criteria were patients with confirmed COVID-19 disease. However, those patients were excluded who were having the history of endocrine diseases or history of renal, liver, heart, or neurological disease. Patients with confirmed Covid-19 were categorized as severe and non-severe according to the clinical characteristics and symptoms and chest radiography results [10].

The study was accepted by the Ethics Committee of the Faculty of Applied Medical Sciences at Taibah University and Saudi Arabia Ministry of Health, General Administration for Research \& Studies.

\subsection{Statistical Analysis}

Data was evaluated using the GraphPad Prism Software (version 5.01). Quantitative data was presented as percentages, mean, standard deviation and range. One-way Anova test was applied to compare between the patient's groups (severe and non-severe). Also, Multivariate analysis was applied based on the Multiple Logistic Regression (severity of COVID-19 infection is dependent parameter tested against the biochemical markers). The odds ratio (OR) was determined with 95\% confidence intervals. All differences were statistically significant at the level of $\mathrm{P} \leq 0.05$ or $\leq 0.001$. Receiver-Operating Characteristic (ROC) curve was created, the Youden's index (J) applied for cut-off values in the diagnosis of COVID-19 and the area under the curve (AUC) was determined by using Epitools - Epidemiological Calculators [11].

\section{Results}

\subsection{Evaluations the Hematological Parameters Levels for Severe and Non-severe Coronavirus Infection}

When compared between two study groups (severe and non-severe), the study indicated that $57.2 \%$ of COVID-19 patients are severe cases and have high mean age $(78 \pm 13.8)$, whereas $42.8 \%$ are non-severe patients with low mean age (54 $\pm 12.01, \quad \mathrm{P}<0.05)$ (Table 1). Comparisons made also according to the severity of infection showed that a statistically significant difference $(\mathrm{P}<0.001)$ was found between the two groups regarding leukocyte, neutrophil and Ferritin values. In patients with severe infection, it was noticed that leukocyte, neutrophil and Ferritin values were higher, whereas lymphocyte was observed to be lower, comparing to non-severe cases (Table 1). While no statistically significant difference was observed between two groups regarding Hemoglobin values $(\mathrm{P}>0.05)$.

Table 1. Hematological markers Characteristics of the Study Patients $(n=112)$, According to Disease Severity.

\begin{tabular}{llll}
\hline Parameters & $\begin{array}{l}\text { Severe group, 64 } \\
(\mathbf{5 7 . 2 \% )}\end{array}$ & $\begin{array}{l}\text { Non-severe group, } \\
\mathbf{4 8}(\mathbf{4 2 . 8 \% )}\end{array}$ & $\boldsymbol{P}$-Value \\
\hline Sex & & & \\
Male & $37(57.8 \%)$ & $25(52.1 \%)$ & \\
Female & $27(42.2 \%)$ & $23(47.9 \%)$ & \\
Age $(\mathrm{Y})$ & $78(13.8)$ & $54(12.01)$ & $0.02^{*}$ \\
Leukocyte $\left(10^{3} / \mathrm{L}\right)$ & $12(3.57)$ & $7.4(2.4)$ & $0.002^{* *}$ \\
Neutrophil $\left(10^{3} / \mathrm{L}\right)$ & $9.9(2.8)$ & $6.7(2.3)$ & $0.01^{*}$ \\
Hemoglobin $(\mathrm{g} / \mathrm{L})$ & $12.5(2.28)$ & $13(1.7)$ & $>0.05$ \\
Platelet $\left(10^{3} / \mathrm{L}\right)$ & $120(20.7)$ & $221(33.6)$ & $0.03^{*}$ \\
Lymphocyte $\left(10^{3} / \mathrm{L}\right)$ & $1.3(9.5)$ & $4.8(9.9)$ & $0.001^{* *}$ \\
S. Ferritin $(\mathrm{ng} / \mathrm{mL})$ & $1857(50.9)$ & $313(24.8)$ & $<0.001^{* *}$ \\
\hline
\end{tabular}

Data presented as number (percentage\%) for sex. One-way Anova test was used to compare between the levels of CBC markers in severe and non-severe Covid-19 infected patients. Data presented as mean (SD), $\mathrm{P}<0.05^{*}, 0.001^{* *}$. The value in bold font has a statistically significant difference.

\subsection{Evaluation the Risk Factors for COVID-19 in Severe Cases}

Multiple regression was applied to evaluate whether age or gender were risk factors and as a result, it was determined that age of the patients was a risk factor for COVID-19 in severe cases ( $\mathrm{P}=0.02$; OR: 2.998; 95\% CI: 0.997-3.802). Whereas 
the patient's gender was not a risk factor for COVID-19 $(\mathrm{P}=0.08$; OR: 0.6598 ; 95\% CI: 0.771-1.322, Data did not show).

Table 2. Multivariate analysis for hematological markers in severe Covid-19 cases.

\begin{tabular}{lllll}
\hline \multirow{2}{*}{ Variables } & \multicolumn{4}{l}{ Multivariate analysis } \\
\cline { 2 - 5 } & $\boldsymbol{r}$ & $\boldsymbol{O R}$ & $\mathbf{9 5 \%}$ CI & $\boldsymbol{P}$ value \\
\hline Leukocyte $\left(10^{3} / \mathrm{L}\right)$ & 0.68 & 4.55 & 2.45 to 6.17 & $0.002^{* *}$ \\
Neutrophil $\left(10^{3} / \mathrm{L}\right)$ & 0.75 & 6.87 & 1.88 to 7.18 & $0.001^{* *}$ \\
Hemoglobin $(\mathrm{g} / \mathrm{L})$ & 0.21 & 1.03 & 0.8930 to 1.055 & 0.57 \\
Platelet $\left(10^{3} / \mathrm{L}\right)$ & 0.12 & 0.59 & 0.07610 to 3.283 & 0.84 \\
Lymphocyte $\left(10^{3} / \mathrm{L}\right)$ & -0.55 & 3.98 & 1.83 to 4.77 & $0.03^{*}$ \\
S. Ferritin $(\mathrm{ng} / \mathrm{mL})$ & 0.53 & 5.88 & 1.00 to 6.01 & $0.01^{*}$ \\
\hline
\end{tabular}

Data presented as $\mathrm{r}=$ Pearson correlation coefficients, $\mathrm{OR}=$ odd ratio, $95 \% \mathrm{CI}=95 \%$ confidence interval. All differences were statistically significant at the level of $P \leq 0.05^{*}$ or $\leq 0.001 * *$.

Furthermore, the Multiple Logistic Regression in which severity was the dependent variable and the hematological markers were the independent variables revealed higher significant odds ratio for leukocytes, neutrophils, and Ferritin (OR: 4.55, OR: 6.87 and OR: 5.88 respectively, Table 2). Also, the associations between severity and the alteration of hematological markers levels were examined by Pearson correlation coefficients (r). The severity of infection associated positively with increasing Leukocyte $(\mathrm{r}=0.68)$, Neutrophil $(\mathrm{r}=0.75)$ and Ferritin $(\mathrm{r}=0.53)$. Whereas a negative association between severity and decreasing of lymphocytes $(\mathrm{r}=-0.55)$ was indicated.

\subsection{Cut-off Values for Significant Indicators in the Prediction of Coronavirus Infection in Severe Cases}

Receiver-Operating Characteristic (ROC) curve was produced, the Youden's index (J) applied for cut-off values in the diagnosis of COVID-19 and the area under the curve (AUC) was calculated. There was a statistically significant variation between severe and non-severe groups according to parameters (leukocyte, neutrophil, Ferritin and lymphocytes), they were then analyzed with ROC analyses. Related to the results yielded by ROC analyses, the following information regarding patients with severe COVID-19 infection were noticed (Table $3 \&$ Figure $1 \mathrm{~A}, \mathrm{~B}, \mathrm{C}$ and D): The cut-off point for leukocyte values was observed to be $\leq 11.2$ (AUC $=0.572$; $\mathrm{p}>0.001 ; 95 \% \mathrm{CI}: 0.465-0.679)$. The cut-off point for neutrophil values was found to be $\leq 10.8$ (AUC $=0.733$; $\mathrm{p}<$ 0.001; 95\% CI: 0.63-0.836). The cut-off point for Ferritin was found to be $\leq 955$ (AUC $=0.937 ; \mathrm{p}<0.001 ; 95 \% \mathrm{CI}$ : $0.886-$ 0.987 ). The cut-off point for lymphocytes values was found to be $\leq 6.9$ (AUC $=0.833 ; \mathrm{p}<0.001 ; 95 \%$ CI: 0.751-0.915) (Table 3 \& Figure 1).

Table 3. Recommended cut-off values for significant markers in the prediction of Coronavirus Disease in severe cases.

\begin{tabular}{llllll}
\hline Parameters & AUC & Cut off & Sensitivity & Specificity & 95\% CI \\
\hline Leukocyte & 0.572 & 11.2 & 0.118 & 0.83 & $0.465-0.679$ \\
Neutrophil & 0.733 & 10.8 & 0.922 & 0.604 & $0.63-0.836$ \\
Lymphocyte & 0.833 & 6.9 & 0.906 & 0.729 & $0.751-0.915$ \\
S. Ferritin & 0.937 & 955 & 0.891 & 0.896 & $<0.001$ \\
\hline
\end{tabular}

Diagnostic cut off values derived from the ROC curve (Receiver operating characteristic curve). AUC: Area under the curve, $95 \% \mathrm{CI}=95 \%$ confidence interval. The values in bold font have a statistically significant difference.
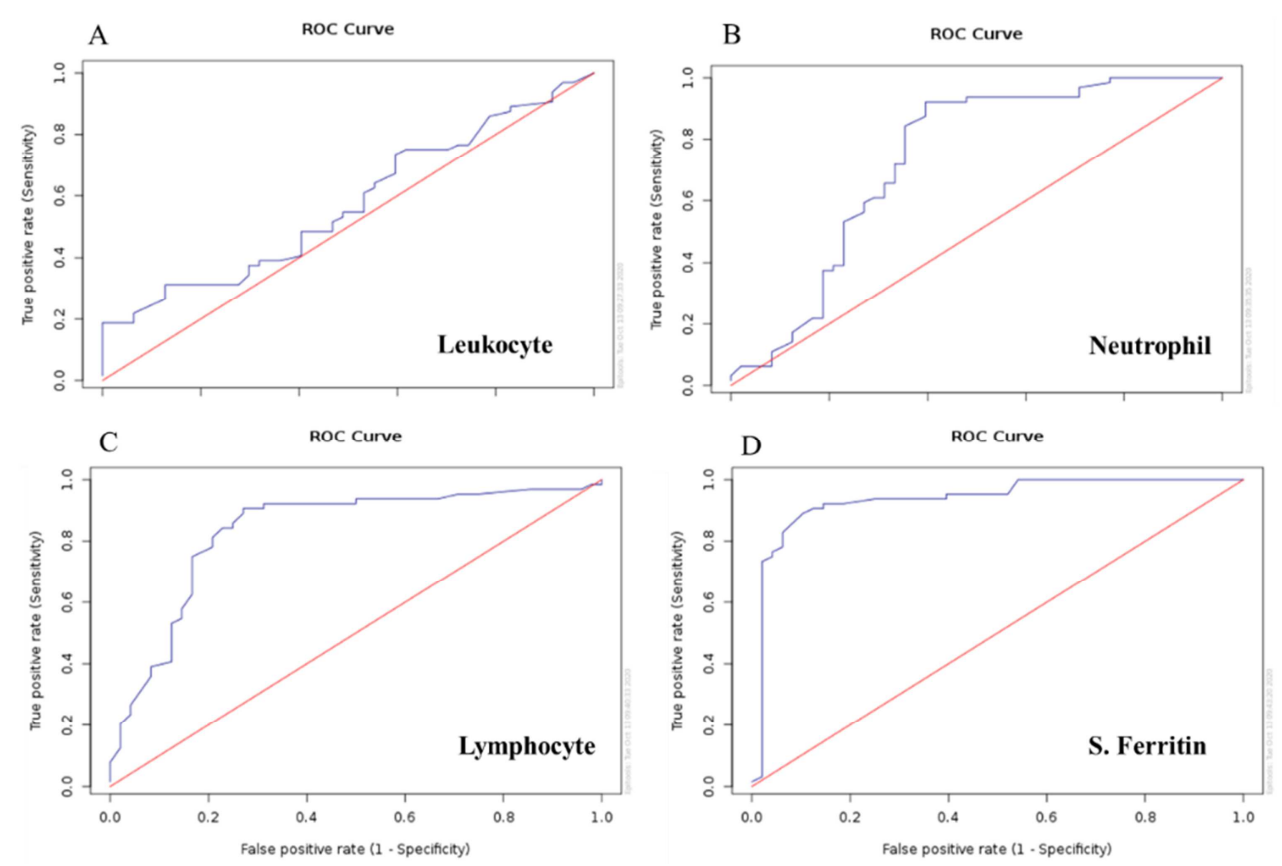

Figure 1. Receiver-operating characteristic curve for significant markers in the prediction of Coronavirus Disease in severe cases. 


\section{Discussion}

The prevalence of COVID-19 infection was enlarged during the last months and distributed overall the world [12]. Consequently, all countries need to evaluate the disease severity and mortality and identify the effective therapy that may limit the spreading of this pandemic [13]. A varied of abnormal laboratory parameters containing biochemical, hematological, inflammatory, and immunological markers considered to be altered during COVID-19 outbreak and lead to the severity of the infection $[14,15]$. In this retrospective study, our team attempted to clarify and determine the various alteration in Ferritin, hematological parameters related to the severity and mortality of COVID-19. The initiation of this study, the investigation different hematological levels were done on 112 patients diagnosed with COVID-19. Current findings indicated that reduction in the number of lymphocytes count but augmentation in the number of leukocytes, neutrophil is directly related to the severity of COVID-19 infection. In patients with the COVID-19 epidemic, the WBC count can vary [16]. Some studies indicated that leukocytosis, and lymphopenia were indicated, and lymphopenia occurred frequently in infected patients $[17,18]$. Additional study advocated that lymphopenia is an efficient marker for the severity of COVID-19 [19], which is confirmed in our study. Furthermore, a retrospective study observed some variations in WBC levels between severe and non-severe COVID-19 patients [20]. Both groups have high count numbers in leucocytes and neutrophil but in the severe group having a considerably greater increase (5.6 vs $\left.4.9 \times 10^{9} / \mathrm{L} ; \mathrm{P}<0.001\right)$. The levels of lymphocytes were less in severe COVID-19 patients $[14,20,21]$ is considered as marker for inflammatory conditions which may be used to indicate disease severity. In the results of our study, which are also consistent with former research, high leukocyte and neutrophil counts and low lymphocytes counts were shown in COVID-19 severe patients. Nevertheless, a bigger study is required to clarify the effectiveness of lymphocytes count as a good marker for early detection of severe COVID-19. In this respect, we may possibly speculate the prospective role of high WBC count with lymphopenia as diagnostic and prognostic marker for severe status of COVID-19.

Concerning the Ferritin levels correlated with the COVID-19 pandemic, this retrospective study detected a higher concentration of Ferritin among severe patients with COVID-19. Recently, a study for 20 patients with COVID-19 found that individuals with severe COVID-19 had increase serum ferritin level, actuality serum ferritin in the severe COVID-19 group significantly greater than in non-severe COVID-19 group (1006.16 $\mathrm{ng} / \mathrm{ml}$ vs $291.13 \mathrm{ng} / \mathrm{ml}$ respectively) [22]. In agreement with this, another study showed that by severe COVID-19 patients, ferritin levels were elevated when admission to the hospital and throughout their staying [23], also the serum ferritin levels exceeded the high limit of detection in these patients until they died.
Furthermore, Chen et al. evaluated the levels of biochemical indicators in 99 patients with COVID-19, indicating elevation in ferritin levels in 12 patients who were died by severe COVID-19 infection [24]. An investigation of the peripheral blood of 69 patients with severe COVID-19 showed raised levels of ferritin compared with patients with non-severe disease, suggesting that serum ferritin levels were strongly correlated to the severity of COVID-19 [25-27]. Our current study showed increased levels of serum ferritin among sever infected patients compared with non-severe patients, and this significant outcome was observed in previous studies. We also indicated a strong association between the elevation of ferritin levels and the severity of COVID-19. In this regard, we may possibly consider the potential role of ferritin as a prognostic biomarker during the severe status of COVID-19. The area under the ROC curve greater than 0.9 has high precision, while $0.7-0.9$ indicates moderate precision, $0.5-0.7$ low precision test [28]. Based on this, Ferritin level was found to be significantly high for patients with severe COVID-19 infection, meaning that it can also be used for diagnosing COVID-19. The cut-off value for Ferritin was observed as being $=955$, and the $\mathrm{AUC}=0.937$ with a sensitivity of $89.1 \%$, and a specificity of $89.6 \%$. Our results also indicated that low lymphocytes levels can probably be used as a diagnostic indicator for COVID-19, because the resulting for lymphocytes were $\mathrm{AUC}=0.833$, cut-off $=6.9$, specificity $=0.729$ and sensitivity $=0.906$. Ferritin is a vital mediator for dysregulation the immune system, particularly under severe hyperferritinemia, through its directly suppressing the immune functions and stimulating the pro-inflammatory factors, causing the cytokine storm [29, $30,31]$. It has been informed that serious outcomes by COVID-19 are associated with cytokine storm syndrome, suggesting that disease severity is related to the cytokine storm syndrome $[5,32]$.

\section{Conclusion}

Our findings supported the hypothesis that ferritin levels may possibly be an important factor affecting the severity of COVID-19. But the limitation of this study was the insufficient number of patients. Studies performed on a larger patient group may obviously identify the importance biomarkers from peripheral blood tests in the diagnosis of COVID-19 patients. However, we supposed that monitoring of ferritin during hospitalization might help to detect severe patients and predict the progression of COVID-19 infection. Several of biochemical and hematological parameters may possibly associate with the severity and mortality of COVID-19 infection and must be examined and evaluated continuously during the progression of this deadly disease. These parameters contained WBCs count, lymphocytes count, and ferritin levels. Because their alterations may lead to vital damage in infected patients and early detection may reduce the progression of COVID-19 severity. 


\section{Funding}

No funding sources.

\section{Competing Interests}

None declared.

\section{Ethical Approval}

The study was approved by the Ethics Committee of the Faculty of Applied Medical Sciences at Taibah University and Saudi Arabia Ministry of Health, General Administration for Research \& Studies.

\section{Author Contributions}

WM and AS: Conceptualization, study design, software, data curation, analysis and interpretation, original draft preparation and formal analysis, reviewing and editing. Author has critically reviewed and approved the final draft and is responsible for the content and similarity index of the manuscript. $\mathrm{HH}$ and MA: Providing the samples, collecting the data. NS: Original draft preparation. Editing language and proof-reading the article. All authors significantly contributed to this research and hold responsibility for its quality and content.

\section{Acknowledgements}

The authors highly appreciate all the hospital team for their efforts and the laboratory staff for data collection of COVID-19.

\section{References}

[1] World Health Organization. Novel coronavirus (2019-nCoV) situation reports.

https://www.who.int/emergencies/diseases/novel-coronavirus2019/situation-reports;2020 [accessed 20 June 2020].

[2] Li Q, Guan X, Wu P, Wang X, Zhou L, Tong Y, et al. Early transmission dynamics in Wuhan, China, of novel coronavirus-infected pneumonia. N Engl J Med 2020; 382: 1199-1207.

[3] Liang W, Guan W, Chen R, et al. Cancer patients in SARS-CoV-2 infection: a nationwide analysis in China. Lancet Oncol 2020; 21: 335.

[4] CDC. COVID-19 Response Team. Preliminary Estimates of the Prevalence of Selected Underlying Health Conditions Among Patients with Coronavirus Disease 2019-United States. MMWR 2020; 69: 382-386.

[5] Zhou F, Yu T, Du R, et al. Clinical course, and risk factors for mortality of adult inpatients with COVID-19 in Wuhan, China: a retrospective cohort study. Lancet 2020; 395: 1054.

[6] Huang C, Wang Y, Li X, et al. Clinical features of patients infected with 2019 novel coronavirus in Wuhan, China. Lancet 2020; 395: 497-506.
[7] Vargas-Vargas M, Cortés-Rojo C. Ferritin levels and COVID-19. Rev Panam Salud Publica 2020; 44: e72.

[8] Wong CK, Lam CW, Wu AK, Ip WK, Lee NL, Chan IH, et al. Plasma inflammatory cytokines and chemokines in severe acute respiratory syndrome. Clin Exp Immunol 2004; 136: 95-103.

[9] Tisoncik JR, Korth MJ, Simmons CP, Farrar J, Martin TR, Katze MG. Into the eye of the cytokine storm. Microbiol Mol Biol Rev 2012; 76: 16-32.

[10] $\mathrm{MOH}$-approved scientific instruction manuals and guidelines for healthcare providers on how to deal with COVID-19 patients. 2020.2 Available from https://www.moh.gov.sa/en/Ministry/MediaCenter/Publication s/Pages/covid19.aspx;2020 [accessed 20 June 2020].

[11] Sergeant. ESG Epitools Epidemiological Calculators. Ausvet. Available at: http://epitools.ausvet.com.au;2020 [accessed 22 August 2020].

[12] Zhou P, Yang XL, Wang XG, Hu B, Zhang L, Zhang W, et al. A pneumonia outbreak associated with a new coronavirus of probable bat origin. Nature 2020; 579: 270-3.

[13] Wang W, Xu Y, Gao R, Lu R, Han K, Wu G, et al. Detection of SARS-CoV-2 in different types of clinical specimens. JAMA 2020; a2020-378.

[14] Rodriguez-Morales AJ. Clinical, laboratory and imaging features of COVID-19: A systematic review and meta-analysis. Travel Med Infect Dis 2020; 34: 101623.

[15] Henry B. M. Hematologic, biochemical, and immune biomarker abnormalities associated with severe illness and mortality in coronavirus disease 2019 (COVID-19): a meta-analysis. Clin Chem Lab Med 2020; 58: 1021-1028.

[16] Di Gennaro F. Coronavirus Diseases (COVID-19) Current Status and Future Perspectives: A Narrative Review. Int J Environ Res Public Health 2020; 17: 2690.

[17] Lippi G., Simundic AM., Plebani M. Potential preanalytical and analytical vulnerabilities in the laboratory diagnosis of coronavirus disease 2019 (COVID-19). Clin Chem Lab Med 2020; 58: 1070-1076.

[18] Lagier JC. Testing the repatriated for SARS-Cov2: Should laboratory-based quarantine replace traditional quarantine? Travel Med Infect Dis 2020; 34: 101624.

[19] Guan W, Ni Z, Hu Y, Liang WH, Ou CQ, He JX, et al. Clinical characteristics of coronavirus disease 2019 in China. N Engl J Med 2020; a2002032.

[20] Lechien JR. Clinical and Epidemiological Characteristics of 1,420 European Patients with mild to-moderate Coronavirus Disease 2019. J Intern Med 2020; 288: 335-344.

[21] Lalueza A, Ayuso B, Arrieta E, Trujillo H, Folgueira D, Cueto C. Elevation of serum ferritin levels for predicting a poor outcome in hospitalized patients with influenza infection. Clin Microbiol Infect 2020; S1198-743: 30100-2.

[22] Fei Zhou, Ting Yu, Ronghui Du, Guohui Fan, Ying Liu, Zhibo Liu, et al. Clinical course and risk factors for mortality of adult inpatients with COVID-19 in Wuhan, China: a retrospective cohort study. Lancet 2020; 395: 1054-62 9. 
[23] Chen N, Zhou M, Dong X, Qu J, Gong F, Han Y, et al. Epidemiological and clinical characteristics of 99 cases of 2019 novel coronavirus pneumonia in Wuhan, China: a descriptive study. Lancet 2020; 395: 507-13.

[24] Fox SE, Akmatbekov A, Harbert JL, Li G, Brown JQ, Vander Heide RS. Pulmonary and Cardiac Pathology in Covid-19: The First Autopsy Series from New Orleans. medRxiv 2020; 04: 06-20050575.

[25] Tao Liu, Jieying Zhang, Yuhui Yang, Hong Ma, Zhengyu Li, Jiaoyu Zhang, et al. The potential role of IL-6 in monitoring severe case of coronavirus disease 2019. medRxiv 2020; 03: 01-20029769.

[26] Mehta P, McAuley DF, Brown M, Sanchez E, Tattersall RS, Manson J. COVID-19: consider cytokine storm syndromes and immunosuppression. Lancet 2020: 6736-306280.

[27] Mandrekar JN. Simple statistical measures for diagnostic accuracy assessment. J Thorac Oncol 2010; 5: 763-764.
[28] Colafrancesco S, Alessandri C, Conti F, Priori R. COVID-19 gone bad: A new character in the spectrum of the hyperferritinemic syndrome? Autoimmun Rev 2020; 19: 102573.

[29] Rosário C, Zandman-Goddard G, Meyron-Holtz EG, D'Cruz DP, Shoenfeld Y. The hyperferritinemic syndrome: macrophage activation syndrome, Still's disease, septic shock and catastrophic antiphospholipid syndrome. BMC Med 2013; 22: 185 .

[30] Wang WK, Chen SY, Liu IJ, Kao CL, Chen HL, Chiang BL, et al. Severe acute respiratory syndrome research Group of the National Taiwan University College of Medicine/NTU Hospital. Temporal relationship of viral load, ribavirin, interleukin (IL)-6, IL-8, and clinical progression in patients with severe acute respiratory syndrome. Clin Infect Dis 2004; 39: 1071-5.

[31] Arentz M. Characteristics and Outcomes of 21 Critically Ill Patients With COVID-19 in Washington State. JAMA 2020; 323: 1612-1614. 